\title{
SISTEMAS DE MANEJO EM PLANTIO DIRETO E SUA INFLUENCIA SOBRE HERBICIDAS APLICADOS EM PÓS-EMERGÊNCIA NA CULTURA DA SOJA
}

\author{
Jamil Constantin ${ }^{1}$, Cleber D. de G. Maciel $^{2}$ e Rubem S. de Oliveira Jr. ${ }^{1}$ \\ ${ }^{1}$ Dr., Professor. Universidade Estadual de Maringá, Depto de Agronomia. Av. Colombo, 5790. Maringá, PR 87020-900 \\ ${ }^{2}$ Eng $^{\circ}$. Agro ., Mestrando. UNESP/FCA, Depto. de Produção Vegetal, Setor Agricultura e Melhoramento Vegetal. Caixa Postal 237. \\ Botucatu, SP18603-970maciel@fca.unesp.br
}

\begin{abstract}
RESUMO
O objetivo do trabalho foi avaliar diferentes sistemas de manejo em plantio direto e o controle em pós-emergência de plantas daninhas na cultura da soja, no município de São João do Ivaí (PR), no ano agrícola de 1998/99. Os tratamentos utilizados nos sistemas seqüenciais de manejo, para o controle de Commelina benghalensis (2 folhas até plantas ramificadas) e Brachiaria plantaginea (2 folhas até plantas perfilhadas), foram: (M1) sulfosate e paraquat/diuron+diquat (864 e $300+240$ $\mathrm{g} / \mathrm{ha}$ ); (M2) sulfosate+2,4-D e paraquat/diuron+diquat ( $864+670$ e 300+240 g/ha); (M3) sulfosate e paraquat/diuron+diquat (672 e 300+240 g/ha); (M4) sulfosate+2,4-D e paraquat/diuron+diquat (672+670 e 300+240 g/ha); (M5) testemunha sem aplicação. A primeira aplicação da seqüencial nos tratamentos M1 e M2 realizou-se aos 26 dias antes da semeadura (DAS), e aos 40 DAS para M3 e M4, e a segunda aplicação no dia da semeadura para todos os tratamentos. Todos os sistemas de manejo foram subdivididos para avaliação do controle de C. benghalensis (1 a 2 folhas) e R. raphanistrum ( 1 a 2 folhas) após a emergência da soja, para os tratamentos com fomesafen ( $125 \mathrm{~g} / \mathrm{ha}$ ) em dose única 19 dias depois da semeadura (DDS), fomesafen e fomesafen ( 125 e $125 \mathrm{~g} / \mathrm{ha})$ em seqüencial aos 19 e $27 \mathrm{DDS}$, chlorimuron-ethyl+imazethapyr $(10+150 \mathrm{~g} / \mathrm{ha}) \mathrm{em}$ dose única 25 DDS e testemunha sem aplicação. A antecipação do manejo, 40 DAS, possibilitou redução no uso de herbicidas e melhor controle de C. benghalensis e B. plantaginea, sendo que, onde o sistema de manejo obteve controle acima de $97 \%$ (M1 e M4), a meia dose de fomesafen (125 g/ha) proporcionou controle satisfatório de C. benghalensis. A seqüência de sulfosate e paraquat/diuron+diquat possibilitou o manejo de C. benghalensis.
\end{abstract}

Palavras chave: Planta daninha, controle, sulfosate, 2,4-D, fomesafen.

\section{ABSTRACT \\ No-tillage management systems and their influence under postemergence herbicides in a soybean crop}

The objective of the experiment was analyse different no-tillage management systems and postemergence control under a soybean crop, in São João do Ivaí (PR), in the agricultural year 1998/99. The treatments used in the management system sequence to control Commelina benghalensis (from 2 leaves to ramified plants) and Brachiaria plantaginea (from 2 leaves to tillered plants) were: (M1) sulfosate and paraquat/diuron+diquat (864 and 300+240 g/ha); (M2) sulfosate+2,4-D and paraquat/diuron+diquat $(864+670$ e 300+240 g/ha); (M3) sulfosate and paraquat/diuron+diquat (672 e $300+240 \mathrm{~g} / \mathrm{ha})$; (M4) sulfosate+2,4-D and paraquat/diuron+diquat $(672+670$ and 300+240 g/ha); (M5) control. The first application of the M1 and M2 sequence was carried out 26 days before sowing (DBS) and at 40 DBS with M3 and M4, and the second application was carried out on the sowing day for all treataments. All manegement systems were subdivided and the control of $C$. benghalensis ( 1 - 2 leaves) and Raphanus raphanistrum (1 - 2 leaves) after soybean emergence was evaluated for the 
Jamil Constantin et al.

treatments with fomesafen (125 g/ha) in a single dose, 19 days after sowing (DAS); fomesafen and fomesafen (125 and 125 $\mathrm{g} / \mathrm{ha})$ in sequence 19 and $27 \mathrm{DAS}$; chlorimuron-ethyl+imazethapyr $(10+150 \mathrm{~g} / \mathrm{ha})$ in a single dose $25 \mathrm{DAS}$, and a control. The antecipation of the management (40 DAS), facilited reduction in herbicide use and a better control of $C$. bengalensis and B. plantaginea. Where the management system obtained control above 97\% (M1 e M4) the half fomesafen dose (125 g/ha) provided a satisfactory control of $C$. benghalensis. The sulfosate and paraquat/diuron+diquat sequences allowed the management of $C$. benghalensis.

Key words: Weed, control, sulfosate, 2,4-D, fomesafen.

\section{INTRODUÇÃO}

O "manejo" em plantio direto, antes do plantio da soja, é fundamental para um bom desenvolvimento da cultura. A eliminação das plantas daninhas antes do plantio permite que a cultura tenha um desenvolvimento inicial livre de interferências. Por isto, o êxito do plantio direto dependerá da disponibilidade de herbicidas que sejam eficazes nas operações de manejo e após a instalação das culturas (Almeida, 1991).

As plantas daninhas possuem baixa capacidade de competir em comunidades instaladas (Piteli, 1990). Desta forma, este conceito justifica a idealização de sistemas que permitam um desenvolvimento da cultura antes da germinação das plantas daninhas. A cultura se estabelecendo primeiro terá um maior poder de competição e, consequentemente, dificultará o estabelecimento das plantas daninhas e ainda aumentará a eficiência dos herbicidas utilizados pois, neste caso, o efeito cultural será um importante aliado, submetendo as invasoras a um ambiente inóspito ao seu desenvolvimento.

Dentre os herbicidas utilizados nas operações de manejo do plantio direto encontram-se o sulfosate, paraquat, diquat, paraquat+diuron, glyphosate e 2,4-D, sendo comum a mistura entre alguns deles (Deuber, 1997; Melhorança et al., 1997; EMBRAPA-CNPSo, 1998 e Rodrigues \& Almeida, 1998).

Trabalhos têm demonstrado que aplicações seqüenciais, onde se aplica primeiro um produto sistêmico como o sulfosate, isolado ou em mistura com 2,4-D, e após 7 a 15 dias, no dia do plantio, aplicam-se produtos de contato como paraquat, paraquat+diuron ou diquat, obtêm uma maior eficácia no controle das plantas daninhas. Isto permite que se plante no limpo, sem plantas daninhas, pois além de eliminar plantas já desenvolvidas os herbicidas também controlam a nova sementeira que porventura possa surgir (Marochi, 1995; Constantin et al., 1997 e Pinto et al., 1997). Já no sistema convencional onde se faz o manejo em dose única de herbicidas, dez dias antes do plantio, os resultados podem não ser satisfatórios além de não controlar a sementeira na hora do plantio, o que pode dificultar o controle das plantas daninhas quando do desenvolvimento da cultura.

Outros trabalhos, em soja, como o de Barros et al. (1997), mostram que a antecipação do manejo feito de forma seqüencial, para 21 dias antes do plantio, pode trazer benefí- cios, como um melhor controle das plantas daninhas pois estas estariam menores, eliminação do primeiro fluxo de germinação das plantas daninhas, possibilidade de substituir o herbicida 2,4-D, que pode ser problemático para culturas sensíveis e, ainda, que a aplicação seqüencial provoca redução significativa do número de plantas daninhas emergidas após o tratamento, em relação a aplicação única dos herbicidas de manejo.

No sistema de plantio direto, o manejo das invasoras é importante não só para que a cultura inicialmente esteja livre das plantas daninhas mas, também, pode ser decisivo para o funcionamento dos herbicidas utilizados durante o estabelecimento da cultura pois, caso não seja eficiente, podem ocorrer rebrotes e intensa reinfestação da área, o que dificultaria a ação de herbicidas utilizados após a emergência da cultura.

O trabalho teve como objetivos avaliar a eficácia de diferentes sistemas de manejo do plantio direto para a cultura da soja e, ainda, verificar a influência destes sistemas na atuação de herbicidas utilizados após a emergência da soja e das plantas daninhas.

\section{MATERIAL E MÉTODOS}

O experimento foi conduzido na Fazenda Santo Antônio, localizada no município de São João do Ivaí, no Estado do Paraná. O semeadura direta foi efetuada em 17/01/99, utilizando-se a cultivar FT-Abyara com espaçamento de 0,45 $\mathrm{m}$ entre linhas e 20 sementes / metro linear.

As aplicações efetuadas antes da semeadura, para eliminar as plantas daninhas e proporcionar à cultura um desenvolvimento sem qualquer interferência, foram denominadas de "manejo". As aplicações de herbicidas efetuadas após a emergência da cultura foram denominadas de "controle na soja ou cultura" ou ainda "controle após a emergência da soja", e visavam o controle de plantas daninhas que germinassem após o manejo ou que escapassem deste.

Inicialmente, para o manejo, pretendia-se dois sistemas onde no primeiro se faria uma aplicação única de sulfosate e sulfosate+2,4-D, de 7 a 10 DAS. (dias antes do semeadura), que seria o padrão. No segundo sistema seria feita uma aplicação seqüencial, onde se anteciparia a primeira aplicação da seqüencial para 20 a 25 DAS e a segunda aplicação 
seria feita no dia da semeadura e, neste caso, se utilizaria o sulfosate e sulfosate $+2,4-D$ na primeira e a mistura em tanque de paraquat/diuron+diquat na segunda aplicação da seqüencial de ambos os tratamentos. A ocorrência de uma estiagem não permitiu o plantio da soja na época prevista. Desta forma, onde a aplicação de manejo ficaria de 7 a 10 DAS e seria única, passou para 26 DAS e acrescentou-se a esta mais uma aplicação de paraquat/diuron+diquat no plantio para se eliminar o mato, passando este sistema, também, para uma aplicação seqüencial. Já nos sistemas previstos inicialmente para serem seqüenciais, as primeiras aplicações que foram inicialmente planejadas para serem de 20 a 25 DAS passaram para 40 DAS pois, somente após 40 dias da primeira aplicação, ocorreram chuvas suficientes para execução da segunda etapa da seqüencial e, consequentemente, desenvolvimento do plantio.

Quando das primeiras aplicações do manejo (07/12/ 98), a infestação era constituída de 22 plantas de trapoeraba (Commelina benghalensis) $/ \mathrm{m}^{2}$ as quais encontravam-se no estádio de 2 folhas até plantas ramificadas, e 49 plantas de capim-marmelada (Brachiaria plantaginea) $/ \mathrm{m}^{2}$ apresentando 2 folhas até plantas perfilhadas. Para o manejo o delineamento experimental utilizado foi o de blocos ao acaso com 5 tratamentos e 4 repetições, sendo as parcelas de $4 \times 20$ metros $\left(80 \mathrm{~m}^{2}\right)$. Em função da estiagem, não houve germinação significativa de novas plantas daninhas, permanecendo o número inicial, constatado em 07/12/98, aproximadamente constante até o plantio.

Após as últimas aplicações dos sistemas de manejo no dia do plantio (17/01/99), subdividiu-se cada parcela em quatro partes. Desta forma, para cada parcela de um sistema de manejo surgiram 4 subparcelas onde realizou-se a aplicação de herbicidas para o controle das plantas daninhas após a emergência da soja. Os tratamentos avaliados foram: fomesafen em dose única, fomesafen em aplicação seqüencial e a mistura de chlorimuron-ethyl+imazethapyr em dose única. Com isto, procurou-se verificar a eficiência destes trata- mentos em função de cada tipo de manejo utilizado anteriormente. As subparcelas, que ficaram sem aplicação de herbicidas após a emergência da soja, só foram utilizadas para auxiliar nas avaliações de controle, sendo retiradas das análises e considerando-se apenas uma testemunha geral. Assim, o delineamento experimental, para avaliação dos herbicidas aplicados após a emergência da soja, foi o de blocos ao acaso com 13 tratamentos e 4 repetições, sendo as parcelas de 4 × $5 \mathrm{~m}\left(20 \mathrm{~m}^{2}\right)$.

O fomesafen em dose única ( $1 / 2$ dose), foi aplicado no dia 05/02/99, ou 19 DDS (dias depois da semeadura), quando haviam 11 plantas de trapoeraba $/ \mathrm{m}^{2}$, estando a maioria no estádio de 1 a 2 folhas, e 44 plantas de nabo (Raphanus raphanistrum) $/ \mathrm{m}^{2}$, com a maioria no estádio de 1 a 2 folhas. A primeira aplicação da seqüencial de fomesafen foi realizada em 05/02/99 (19 DDS) e a segunda em 13/02/99 (27 DDS), e as condições das plantas daninhas eram as mesmas que para o fomesafen em dose única. A mistura chlorimuronethyl+imazethapyr foi aplicada em dose única em 11/02/99 (25 DDS), sendo que o número e as espécies de plantas daninhas eram os mesmos descritos anteriormente, mas apresentando estágio de 2 a 6 folhas. Desta forma, observa-se que após a emergência da soja germinaram apenas trapoeraba e nabo, e que o nabo não se fazia presente por ocasião do manejo.

Na Tabela 1 encontram-se o nome comum, nome comercial e formulação dos herbicidas utilizados no experimento; na Tabela 2 estão os tratamentos, doses e épocas de aplicação utilizados no manejo antes do plantio e na Tabela 3 estão os tratamentos, doses e épocas de aplicação dos herbicidas utilizados para o controle de plantas daninhas após a emergência da soja.

Em todas as aplicações, de manejo ou controle após a emergência da soja, utilizou-se um pulverizador costal de pressão constante a base de $\mathrm{CO}_{2}$, com bico tipo leque 110-SF02 e pressão de $2,0 \mathrm{kgf} / \mathrm{cm}^{2}$, o que proporcionou um volume de 200 l/ha de calda. Nas aplicações a velocidade do vento

Tabela 1. Características dos herbicidas utilizados no experimento. São João do Ivaí, PR. 1998/ 99.

\begin{tabular}{lccc}
\hline \multirow{2}{*}{ Nome comum } & \multirow{2}{*}{ Nome comercial } & \multicolumn{2}{c}{ Formulação } \\
\cline { 3 - 4 } Sulfosate & Zapp & Tipo & Concentração Total \\
Paraquat/diuron & Gramocil & S.C. & $480 \mathrm{~g} / 1$ \\
Diquat & Reglone & Sol. Aq. C. & $200 \mathrm{~g} / 1 / 100 \mathrm{~g} / 1$ \\
$2,4-D$ & DMA 806 BR & Sol. Aq. C. & $200 \mathrm{~g} / 1$ \\
Fomesafen & Flex & Sol. Aq. C. & $806 \mathrm{~g} / 1$ \\
Imazethapyr & Pivot & Sol. Aq. C. & $250 \mathrm{~g} / 1$ \\
Chlorimuron-ethyl & Classic & Gr.D.A. & $100 \mathrm{~g} / 1$ \\
\hline
\end{tabular}


Jamil Constantin et al.

Tabela 2. Tratamentos, doses e épocas de aplicação seqüencial nos diferentes sistemas de manejo das plantas daninhas antes do plantio da soja. São João do Ivaí, PR. 1998/ 99.

\begin{tabular}{lcc}
\hline \multicolumn{1}{c}{ Tratamento } & $\begin{array}{c}\text { Dose } \\
\text { (g/ha) }\end{array}$ & Época de Aplicação \\
\hline M1 = sulfosate e paraquat/diuron+diquat & 864 e $300+240$ & 26 DAS e no dia do plantio \\
M2 = sulfosate+2,4-D e paraquat/diuron+diquat & $864+670$ e $300+240$ & 26 DAS e no dia do plantio \\
M3 = sulfosate e paraquat/diuron+diquat & 672 e $300+240$ & 40 DAS e no dia do plantio \\
M4 = sulfosate+2,4-D e paraquat/diuron+diquat & $672+670$ e $300+240$ & 40 DAS e no dia do plantio \\
Testemunha & - & - \\
\hline
\end{tabular}

OBS: - DAS = Dias antes da semeadura.

- As aplicações realizadas no dia do plantio foram feitas imediatamente antes da semeadura, ou seja, no sistema aplique e plante.

Tabela 3. Tratamentos, doses e épocas de aplicação dos herbicidas utilizados no controle bde plantas daninhas após a emergência da cultura e da nova infestação, em complemento aos diferentes manejo utilizados antes do plantio da soja. São João do Ivaí, PR. 1998/ 99.

\begin{tabular}{lcc}
\hline \multicolumn{1}{c}{ Tratamentos } & $\begin{array}{c}\text { Dose } \\
\text { (g/ha) }\end{array}$ & $\begin{array}{c}\text { Época de } \\
\text { Aplicação (DDS) }\end{array}$ \\
\hline M1 = fomesafen (Dose única 1/2) & 125 & 19 \\
M1 = fomesafen e fomesafen (Seqüencial) & 125 e 125 & 19 e 27 \\
M1 = chlorimuron-ethyl+imazethapyr (Dose única) & $10+150$ & 25 \\
M2 = fomesafen (Dose única 1/2) & 125 & 19 \\
M2 = fomesafen e fomesafen (Seqüencial) & 125 e 125 & 19 e 27 \\
M2 = chlorimuron-ethyl+imazethapyr (Dose única) & $10+150$ & 25 \\
M3 = fomesafen (Dose única 1/2) & 125 & 19 \\
M3 = fomesafen e fomesafen (Seqüencial) & 125 e 125 & 19 e 27 \\
M3 = chlorimuron-ethyl+imazethapyr (Dose única) & $10+150$ & 25 \\
M4 = fomesafen (Dose única 1/2) & 125 & 19 \\
M4 = fomesafen e fomesafen (Seqüencial) & 125 e 125 & 19 e 27 \\
M4 = chlorimuron-ethyl+imazethapyr (Dose única) & $10+150$ & 25 \\
Testemunha & - & - \\
\hline
\end{tabular}

OBS: - DDS = Dias depois da semeadura.

- M1, M2, M3 e M4 = correspondem aos diferentes sistemas de manejo utilizados antes do plantio.

não ultrapassou $4 \mathrm{~km} / \mathrm{h}$, a umidade relativa foi sempre superior a $70 \%$ e as plantas daninhas não estavam em condição de estresse.

As avaliações de percentagem de controle (0-100\%) das plantas daninhas foram efetuadas aos 0, 7 e 11 DDS (dias depois da semeadura) para os tratamentos de manejo, e aos 26, 33 e 47 DDS para os tratamentos efetuados após a emergência da cultura da soja. Para melhor visualização dos resultados, os dados de controle de plantas daninhas após a emergência da cultura foram analisados separadamente, estudando-se cada tratamento dentro dos diferentes tipos de manejo, e em conjunto, onde foram comparados todos os tratamentos dentro dos diferentes tipos de manejo.
Os dados obtidos foram submetidos análise de variância pelo teste $\mathrm{F}$ e as médias foram comparadas pelo teste de Tukey a nível de $5 \%$ de probabilidade.

\section{RESULTADOS E DISCUSSÃO}

\section{Manejo}

Os dados de controle para os diferentes sistemas de manejo de trapoeraba aos 0,7 e 11 DDS encontram-se na Tabela 4. Verifica-se que no dia do plantio apenas o sistema 
Tabela 4. Efeito dos sistemas de manejo (aplicação sequencial), realizados antes do plantio da soja, em percentagem de controle de Commelina benghalensis e Brachiaria plantaginea aos 0, 7 e 11 DDS. São João do Ivaí, PR. 1998/ 99. (Média de quatro repetições - Dados originais).

\begin{tabular}{|c|c|c|c|c|c|c|c|}
\hline \multirow{2}{*}{ Tratamento } & \multirow{2}{*}{$\begin{array}{c}\text { Dose } \\
\text { (g/ha) }\end{array}$} & \multicolumn{3}{|c|}{ Commelina benghalensis } & \multicolumn{3}{|c|}{ Brachiaria plantaginea } \\
\hline & & 0 DDS & 7 DDS & 11 DDS & 0 DDS & 7 DDS & 11 DDS \\
\hline $\mathrm{Ml}=$ sulfosate $\mathrm{e}$ paraquat/diuron+diquat & 864 e $300+240$ & $42 \mathrm{c}$ & $92 \mathrm{bc}$ & $87 \mathrm{~b}$ & $94 \mathrm{a}$ & 99 a & $97 \mathrm{~b}$ \\
\hline $\mathrm{M} 2=$ sulfosate $+2,4-\mathrm{D}$ e paraquat/diuron + diquat & $864+670$ e $300+240$ & $66 \mathrm{~b}$ & $96 \mathrm{ab}$ & $97 \mathrm{a}$ & $87 \mathrm{~b}$ & $99 \mathrm{a}$ & $96 \mathrm{~b}$ \\
\hline $\mathrm{MB}=$ sulfosate e paraquat/diuron+diquat & 672 e $300+240$ & $41 \mathrm{c}$ & $89 c$ & $85 b$ & 99 a & $100 \mathrm{a}$ & $100 \mathrm{a}$ \\
\hline $\mathrm{M} 4=$ sulfosate $+2,4-\mathrm{D}$ e paraquat/diuron + diquat & $672+670$ e $300+240$ & $91 \mathrm{a}$ & $98 \mathrm{a}$ & $100 \mathrm{a}$ & $95 \mathrm{a}$ & $100 \mathrm{a}$ & $98 \mathrm{ab}$ \\
\hline Testemunha & - & $0 \mathrm{~d}$ & $0 \mathrm{~d}$ & $0 \mathrm{c}$ & $0 \mathrm{c}$ & $0 \mathrm{~b}$ & $0 \mathrm{c}$ \\
\hline $\mathrm{F}^{*}$ & - & 58,0 & 1582,5 & 984,7 & 1350,9 & 14852,2 & 6032,2 \\
\hline C.V. (\%) & - & 18,4 & 2,8 & 3,6 & 3,1 & 0,9 & 1,4 \\
\hline D.M.S. (5\%) & - & 20,0 & 4,8 & 6,0 & 5,2 & 1,6 & 2,5 \\
\hline
\end{tabular}

OBS: - DDS= Dias depois da semeadura.

- Médias, na mesma coluna, seguidas da mesma letra não diferem significativamente entre si.

$-*(\mathrm{p}<0,05)$.

- M1 e M2 = A primeira aplicação da seqüencial aos 26 DAS e a segunda no dia da semeadura.

- M3 e M4 = A primeira aplicação da seqüencial aos 40 DAS e a segunda no dia da semeadura.

M4 obteve um bom controle, sendo que os demais ainda não haviam conseguido uma eficácia satisfatória neste período. A partir dos 7 DDS todos os sistemas conseguiram controle satisfatório, em função da aplicação no plantio de paraquat/ diuron+diquat ter demonstrado seu efeito complementar à ação das primeiras aplicações. Aos 11 DDS, os tratamentos M2 e M4 obtiveram excelentes níveis de controle, sendo significativamente superiores aos tratamentos M1 e M3, os quais alcançaram percentagem de controle em torno de $86 \%$. Observa-se que as aplicações efetuadas 40 DAS tenderam a ser superiores aos manejos onde as aplicações foram efetuadas aos $26 \mathrm{DAS}$, principalmente nos manejos onde havia a presença de 2,4-D. Assim, o tratamento M4, mesmo tendo $20 \%$ a menos de sulfosate, conseguiu maiores índices de controle que o tratamento M2. Isto provavelmente é explicado porque aos 40 DAS as plantas daninhas apresentavam-se menores e mais sensíveis a ação dos herbicidas. O que demonstra ser vantajosa a antecipação do manejo, tanto em termos de controle como em termos de economia. Pode-se observar ainda que onde se utilizou a mistura de sulfosate $+2,4-\mathrm{D}$ o controle de trapoeraba foi superior aos demais tratamentos. Para o capim-marmelada (Tabela 4) todos os sistemas demonstraram excelentes níveis de controle ( $\geq 96 \%$ aos 11 DDS), sendo que os manejos efetuados aos 40 DAS também apresentaram-se superiores aos 11 DDS, ressaltando novamente a maior eficácia do controle precoce.

\section{Controle após a emergência da soja}

$\mathrm{Na}$ Tabela 5, pode-se observar que a meia dose de fomesafen apresentou-se eficiente no controle de trapoeraba para todos os sistemas de manejo aos 26 DDS, sendo que a partir dos 33 DDS os sistemas M1 e M3 não conseguiram controle satisfatório e nos sistemas M2 e M4 o controle para a trapoeraba apresentou-se em torno de $85 \%$ até os 47 DDS. Isto demonstra ser perfeitamente viável a utilização do fomesafen em meia dose, desde que no manejo o controle da trapoeraba tenha uma eficácia elevada ( $\geq 97 \%$ ), pois a constatação da redução de controle da meia dose de fomesafen nos sistemas M1 e M3 foi resultante dos rebrotes de trapoeraba que escaparam ao manejo e não àquelas que germinaram após o plantio.

A seqüencial de fomesafen proporcionou controle satisfatório da trapoeraba aos 33 e 47 DDS em todos os sistemas de manejo (Tabela 5). Apesar disto, nos sistemas M2 e M4 os níveis de controle foram significativamente superiores aos obtidos pela seqüencial de fomesafen nos sistemas M1 e M3, os quais obtiveram no manejo níveis de controle de trapoeraba de 87 e $85 \%$, respectivamente.

Para a mistura de chlorimuron-ethyl+imazethapyr aos 33 DDS a ação dos herbicidas, que são sistêmicos, ainda não havia se realizado (Tabela 5). Já aos 47 DDS, os níveis de controle da trapoeraba foi considerado satisfatório, princi- 
Tabela 5. Efeito da meia dose e seqüencial de fomesafen e da mistura de chlorimuron-ethyl+imazethapyr em complemento aos diferentes manejos, aplicadas após a emergência da soja, em percentagem de controle de Commelina benghalensis. São João do Ivaí, PR. 1998/99. (Média de quatro repetições - Dados originais).

\begin{tabular}{|c|c|c|c|c|}
\hline \multirow[t]{2}{*}{ Tratamentos } & \multirow{2}{*}{$\begin{array}{l}\text { Dose } \\
\text { (g/ha) }\end{array}$} & \multicolumn{3}{|c|}{ Commelina benghalensis } \\
\hline & & $26 \mathrm{DDS}$ & $33 \mathrm{DDS}$ & 47 DDS \\
\hline M 1= fomesafen (Dose única $1 / 2)$ & 125 & $88 \mathrm{ab}$ & $60 \mathrm{ab}$ & $51 \mathrm{~b}$ \\
\hline $\mathrm{M} 2=$ fomesafen $($ Dose única $1 / 2)$ & 125 & $96 \mathrm{a}$ & $87 \mathrm{a}$ & $87 \mathrm{a}$ \\
\hline $\mathrm{M} 3=$ fomesafen (Dose única $1 / 2)$ & 125 & $86 \mathrm{~b}$ & $57 \mathrm{~b}$ & $54 \mathrm{~b}$ \\
\hline $\mathrm{M} 4=$ fomesafen (Dose única $1 / 2$ ) & 125 & $95 \mathrm{ab}$ & $88 \mathrm{a}$ & $93 \mathrm{a}$ \\
\hline Testemunha & - & $0 \mathrm{c}$ & $0 \mathrm{c}$ & $0 \mathrm{c}$ \\
\hline $\mathrm{F}$ & - & $330,4 *$ & $30,7^{*}$ & $30,4^{*}$ \\
\hline C.V. $(\%)$ & - & 6,2 & 22,0 & 23,6 \\
\hline \multirow[t]{2}{*}{ D.M.S. $(5 \%)$} & - & 10,2 & 29,0 & 30,3 \\
\hline & & $26 \mathrm{DDS}$ & 33 DDS & 47 DDS \\
\hline M1 = fomesafen e fomesafen (Seqüêncial) & 125 e 125 & - & $86,3 \mathrm{~b}$ & $83 \mathrm{~b}$ \\
\hline M2= fomesafen e fomesafen (Seqüêncial) & 125 e 125 & - & $95 \mathrm{a}$ & $92 \mathrm{a}$ \\
\hline M3 = fomesafen e fomesafen (Seqüêncial) & 125 e 125 & - & $80 \mathrm{c}$ & $84 \mathrm{~b}$ \\
\hline M4 = fomesafen e fomesafen (Seqüêncial) & 125 e 125 & - & $97 \mathrm{a}$ & $94 \mathrm{a}$ \\
\hline Testemunha & - & - & $0 \mathrm{~d}$ & $0 \mathrm{c}$ \\
\hline $\mathrm{F}$ & - & - & $1183,9 *$ & $478,9^{*}$ \\
\hline C.V. $(\%)$ & - & - & 3,3 & 5,1 \\
\hline \multirow[t]{2}{*}{ D.M.S. $(5 \%)$} & - & - & 5,3 & 8,2 \\
\hline & & $26 \mathrm{DDS}$ & $33 \mathrm{DDS}$ & $47 \mathrm{DDS}$ \\
\hline M1= chlorimuron+imazethapyr (Dose única) & $10+150$ & - & $36 \mathrm{~b}$ & $82 \mathrm{~b}$ \\
\hline M2 $=$ chlorimuron+imazethapyr (Dose única) & $10+150$ & - & $49 \mathrm{~b}$ & $96 \mathrm{a}$ \\
\hline M3 = chlorimuron+imazethapyr (Dose única) & $10+150$ & - & $48 \mathrm{~b}$ & $89 \mathrm{ab}$ \\
\hline M4 = chlorimuron+imazethapyr (Dose única) & $10+150$ & - & $66 \mathrm{a}$ & $97 \mathrm{a}$ \\
\hline Testemunha & - & - & $0 \mathrm{c}$ & $0 \mathrm{c}$ \\
\hline $\mathrm{F}$ & - & - & $63,1^{*}$ & $448,0^{*}$ \\
\hline C.V. $(\%)$ & - & - & 15,6 & 5,1 \\
\hline D.M.S. $(5 \%)$ & - & - & 14,0 & 8,4 \\
\hline
\end{tabular}

OBS: - DDS = dias depois da semeadura.

- Médias, na mesma coluna, seguidas da mesma letra não diferem significativamente entre si.

$-*(\mathrm{p}<0,05)$.

- A meia dose de fomesafen foi aplicado aos 19 DDS. 
Tabela 6. Efeito dos tratamentos aplicados após a emergência da soja, em complemento aos diferentes tipos de manejo, em percentagem de controle de Commelina benghalensis. São João do Ivaí, PR. 1998/99. (Média de quatro repetições - Dados originais).

\begin{tabular}{|c|c|c|c|}
\hline \multirow[t]{2}{*}{ Tratamento } & \multirow{2}{*}{$\begin{array}{c}\text { Dose } \\
(g / h a)\end{array}$} & \multicolumn{2}{|c|}{ Commelina benghalensis } \\
\hline & & 33 DDS & $47 \mathrm{DDS}$ \\
\hline M1 = fomesafen (Dose única $1 / 2)$ & 125 & $60 \mathrm{~cd}$ & $51 \mathrm{~b}$ \\
\hline M1 = fomesafen e fom esafen (Seqüencial) & 125 e 125 & $86 \mathrm{ab}$ & 83 a \\
\hline M1 = chlorimuron-ethyl+imazethapyr (Dose única) & $10+150$ & $36 \mathrm{e}$ & $82 \mathrm{a}$ \\
\hline M2 = fomesafen $($ Dose única $1 / 2)$ & 125 & $87 \mathrm{ab}$ & $87 \mathrm{a}$ \\
\hline M2 = fomesafen e fom esafen (Seqüencial) & 125 e 125 & $95 \mathrm{a}$ & 92 a \\
\hline $\mathrm{M} 2=$ chlorimuron-ethyl+imazethapyr (Dose única) & $10+150$ & $49 \mathrm{de}$ & $96 \mathrm{a}$ \\
\hline M3 = fomesafen $($ Dose única $1 / 2)$ & 125 & $57 \mathrm{de}$ & $54 \mathrm{~b}$ \\
\hline M3 = fom esafen e fom esafen (S eqüencial) & 125 e 125 & $80 \mathrm{abc}$ & $84 \mathrm{a}$ \\
\hline M3 = chlorimuron-ethyl+imazethapyr (Dose única) & $10+150$ & $48 \mathrm{de}$ & 89 a \\
\hline M4 = fomesafen $($ Dose única $1 / 2)$ & 125 & $88 \mathrm{ab}$ & 93 a \\
\hline M4 = fom esafen e fom esafen (Seqüencial) & 125 e 125 & $97 \mathrm{a}$ & $94 \mathrm{a}$ \\
\hline M4 $=$ chlorimuron-ethyl+imazethapyr (Dose única) & $10+150$ & $66 \mathrm{bcd}$ & $97 \mathbf{a}$ \\
\hline Testemunha & - & $0 \mathrm{f}$ & $0 \mathrm{c}$ \\
\hline $\mathrm{F}$ & - & $39,0^{*}$ & $38,4^{*}$ \\
\hline C.V. $(\%)$ & - & 13,6 & 11,5 \\
\hline D.M.S. $(5 \%)$ & - & 22,4 & 22,2 \\
\hline
\end{tabular}

OBS: - DDS = dias depois da semeadura.

- Médias, na mesma coluna, seguidas da mesma letra não diferem significativamente entre si.

$-*(\mathrm{p}<0,05)$.

- M1, M2, M3 e M4 = diferentes sistemas de manejo utilizados antes da semeadura.

palmente nos manejos M2 e M4, os quais foram superiores ao chlorimuron-ethyl+imazethapyr nos sistemas de manejo M1 e M3. Ressalte-se que no sistema M1 o controle ficou no limite inferior aceitável (82\%), igualando-se ao sistema M3, mas sendo inferior significativamente aos sistemas M2 e M4. Já o chlorimuron-ethyl+imazethapyr no sistema M3 não diferiu significativamente dos sistemas M2 e M4, demonstrando que mesmo tendo $20 \%$ a menos de sulfosate, o sistema M3, aplicado aos 40 DAS, conseguiu melhorar a eficácia dos herbicidas aplicados em pós-emergência.

$\mathrm{Na}$ Tabela 6 encontra-se uma análise conjunta dos resultados de controle da trapoeraba aos 33 e 47 DDS para todos os tratamentos aplicados após a emergência da soja. Nesta análise, pode-se observar que houve um aumento no coeficiente de variação e consequentemente nas diferenças mínimas significativas, o que impossibilitou a distinção das diferenças detectadas quando se analisou separadamente a seqüencial de fomesafen e a mistura de chlorimuronethyl+imazethapyr. Assim, apenas a meia dose de fomesafen nos sistemas de manejo M1 e M3 não obteve controle satisfatório da trapoeraba, sendo significativamente inferior aos demais tratamentos. Para este conjunto de informações, pode-se notar que os maiores níveis de controle dos herbicidas aplicados após a emergência da soja foram para os sistemas onde o manejo foi realizado aos 40 DAS, quando se compa- ram M1 com M3 e M2 com M4, onde nos tratamentos utilizaram-se os mesmos produtos.

Para o nabo os resultados encontram-se nas Tabelas 7 e 8. Verifica-se que para esta espécie, independente dos sistema de manejo utilizado, o controle para os tratamentos após a emergência da cultura foram excelentes, não havendo diferenças significativas entre os mesmos em nenhuma das avaliações.

Os resultados permitem considerar que o sistema de manejo utilizado antes do plantio foi decisivo para bom funcionamento dos herbicidas aplicados após a emergência da soja. E isto se deve, principalmente, em função de plantas que escapam ao manejo e posteriormente rebrotam junto com a cultura, dificultando a ação dos herbicidas aplicados após a emergência da soja, uma vez que estes são mais específicos para plantas germinadas do que para plantas rebrotadas. Este fato pode ser constatado, neste experimento, para o controle de trapoeraba.

Além disto, também pode-se constatar que onde o controle da trapoeraba no manejo apresentou-se igual ou superior a $97 \%$, a meia dose de fomesafen obteve índice de controle satisfatório. No entanto, onde o manejo não obteve este índice elevado, apenas a seqüencial de fomesafen e a mistura de chlorimuron-ethyl+imazethapyr conseguiram controle satisfatório. O uso do 2,4-D no manejo melhorou o controle 
Tabela 7. Efeito da meia dose e seqüencial de fomesafen e da mistura de chlorimuron-ethyl em complemento aos diferentes manejos, aplicadas após a emergência da soja, em percentagem de controle de Raphanus raphanistrum. São João do Ivaí, PR. 1998/99. (Média de quatro repetições - Dados originais).

\begin{tabular}{|c|c|c|c|c|}
\hline \multirow[t]{2}{*}{ Tratamentos } & \multirow{2}{*}{$\begin{array}{l}\text { Dose } \\
\text { (g/ha) }\end{array}$} & \multicolumn{3}{|c|}{ Raphanus raphanistrum } \\
\hline & & $26 \mathrm{DDS}$ & 33 DDS & 47 DDS \\
\hline M1 = fomesafen (Dose única $1 / 2)$ & 125 & 100 & 100 & $100 \mathrm{a}$ \\
\hline $\mathrm{M} 2=$ fomesafen (Dose única $1 / 2)$ & 125 & 100 & 100 & $100 \mathrm{a}$ \\
\hline $\mathrm{M} 3=$ fomesafen $(\mathrm{D}$ ose única $1 / 2)$ & 125 & 100 & 100 & $98 \mathrm{a}$ \\
\hline $\mathrm{M} 4=$ fomesafen $(\mathrm{D}$ ose única $1 / 2)$ & 125 & 100 & 100 & $99 \mathrm{a}$ \\
\hline Testemunha & - & 0 & 0 & $0 \mathrm{~b}$ \\
\hline $\mathrm{F}$ & - & - & - & $6086,4^{*}$ \\
\hline C.V. $(\%)$ & - & - & - & 1,43 \\
\hline \multirow[t]{2}{*}{ D.M.S. $(5 \%)$} & - & - & - & 2,57 \\
\hline & & 26 DDS & 33 DDS & $47 \mathrm{DDS}$ \\
\hline M1 = fomesafen e fomesafen (Seqüencial) & 125 e 125 & - & 100 & 100 \\
\hline M2 = fomesafen e fomesafen (Seqüencial) & 125 e 125 & - & 100 & 100 \\
\hline M3 = fomesafen e fomesafen (Seqüencial) & 125 e 125 & - & 100 & 100 \\
\hline M4 = fomesafen e fomesafen (Seqüencial) & 125 e 125 & - & 100 & 100 \\
\hline \multirow[t]{2}{*}{ Testemunha } & - & - & 0 , & 0 \\
\hline & & $26 \mathrm{DDS}$ & 33 DDS & 47 DDS \\
\hline M1 = chlorimuron + imazethapyr (Dose única) & $10+150$ & - & $97 \mathrm{a}$ & 100 \\
\hline M2 = chlorimuron + imazethapyr (Dose única) & $10+150$ & - & $96 \mathrm{a}$ & 100 \\
\hline M3 = chlorimuron + imazethapyr (Dose única) & $10+150$ & - & $96 \mathrm{a}$ & 100 \\
\hline M4 = chlorimuron + imazethapyr (Dose única) & $10+150$ & - & 93 a & 100 \\
\hline Testemunha & - & - & $0 \mathrm{~b}$ & 0 \\
\hline$F$ & - & - & $688,0^{*}$ & - \\
\hline C.V. $(\%)$ & - & - & 4,3 & - \\
\hline D.M.S. $(5 \%)$ & - & - & 7,3 & - \\
\hline
\end{tabular}

OBS: - DDS $=$ dias depois da semeadura.

- Médias, na mesma coluna, seguidas da mesma letra não diferem significativamente entre si.

$-^{*}(\mathrm{p}<0,05)$.

- A meia dose de fomesafen foi aplicada aos 19 DDS.

da trapoeraba pelos herbicidas aplicados após a emergência da soja.

A antecipação do manejo permitiu que doses menores do herbicida sulfosate pudessem funcionar melhor que doses maiores de sulfosate, aplicadas 14 dias depois. E isto se deve ao estágio das plantas daninhas pois, quando se ante- cipa o manejo, as plantas daninhas estão menores e mais sensíveis.

O experimento, no manejo, passou por um período de estiagem e os sistemas de manejo previstos inicialmente para serem mais antecipados, mostraram-se mais seguros e econômicos. 
Tabela 8. Efeito dos tratamentos aplicados após a emergência da soja, em complemento aos diferentes tipos de manejo, em percentagem de controle de Raphanus raphanistrum. São João do Ivaí, PR. 1998/99 (Média de quatro repetições - Dados originais).

\begin{tabular}{lccc}
\hline \multicolumn{1}{c}{ Tratamentos } & Dose & \multicolumn{2}{c}{ Raphanus raphanistrum } \\
\cline { 3 - 4 } & $(\mathrm{g} / \mathrm{ha})$ & 33 DDS & 47 DDS \\
\hline M1 = fomesafen (Dose única 1/2) & 125 & $100 \mathrm{a}$ & $100 \mathrm{a}$ \\
M1 = fomesafen e fomesafen (Seqüencial) & $125 \mathrm{e} 125$ & $100 \mathrm{a}$ & $100 \mathrm{a}$ \\
M1 = chlorimuron-ethyl + imazethapyr (Dose única) & $10+150$ & $97 \mathrm{ab}$ & $100 \mathrm{a}$ \\
M2 = fomesafen (Dose única 1/2) & 125 & $100 \mathrm{a}$ & $100 \mathrm{a}$ \\
M2 = fomesafen e fomesafen (Seqüencial) & $125 \mathrm{e} 125$ & $100 \mathrm{a}$ & $100 \mathrm{a}$ \\
M2 = chlorimuron-ethyl + imazethapyr (Dose única) & $10+150$ & $96 \mathrm{ab}$ & $100 \mathrm{a}$ \\
M3 = fomesafen (Dose única 1/2) & 125 & $100 \mathrm{a}$ & $98 \mathrm{a}$ \\
M3 = fomesafen e fomesafen (Seqüencial) & $125 \mathrm{e} 125$ & $100 \mathrm{a}$ & $100 \mathrm{a}$ \\
M3 = chlorimuron-ethyl + imazethapyr (Dose única) & $10+150$ & $96 \mathrm{ab}$ & $100 \mathrm{a}$ \\
M4 = fomesafen (Dose única 1/2) & 125 & $100 \mathrm{a}$ & $99 \mathrm{a}$ \\
M4 = fomesafen e fomesafen (Seqüencial) & $125 \mathrm{e} 125$ & $100 \mathrm{a}$ & $100 \mathrm{a}$ \\
M4 = chlorimuron-ethyl + imazethapyr (Dose única) & $10+150$ & $93 \mathrm{~b}$ & $100 \mathrm{a}$ \\
Testemunha & - & $0 \mathrm{c}$ & $0 \mathrm{~b}$ \\
\hline F & - & $615,3^{*}$ & $6080,8^{*}$ \\
C.V.(\%) & - & 2,4 & 0,8 \\
D.M.S. (5\%) & - & 5,5 & 1,8 \\
\hline
\end{tabular}

OBS: - DDS = dias depois da semeadura.

- Médias, na mesma coluna, seguidas da mesma letra não diferem significativamente entre si.

- * $(\mathrm{p}<0,05)$.

- M1, M2, M3 e M4 = diferentes sistemas de manejo utilizados antes da semeadura.

\section{CONCLUSÕES}

A antecipação do manejo em condição de estiagem, 40 DAS, permitiu uma redução no uso de herbicidas e um melhor controle de Commelina benghalensis e Brachiaria plantaginea.

Apesar de todos sistemas de manejo obterem um controle satisfatório de Commelina benghalensis, a utilização de 2,4-D proporcionou maior eficácia.

É possível se fazer manejo da Commelina benghalensis sem 2,4-D e obter-se um controle satisfatório com a seqüencial de sulfosate e paraquat/diuron+diquat.

O sistema de manejo foi decisivo no funcionamento dos herbicidas aplicados após a emergência da soja.

\section{LITERATURA CITADA}

ALMEIDA, F.S. Controle de plantas daninhas em plantio direto. Londrina: IAPAR, 1991. 34p. (IAPAR. Circular, 67).

BARROS, A. C. et al. Desempenho de sulfosate com ou na ausência de 2,4-D e a seqüencial de paraquat ou diquat na dessecação da vegetação daninha. In: CONGRES-
SO BRASILEIRO DA CIÊNCIA DAS PLANTAS DANINHAS, 21, 1997, Caxambú. Resumos... Caxambú: SBCPD, 1997. p.55.

CONSTANTIN, J. et al. Eficiência e efeito residual do herbicida sulfosate no controle de Ipomoea grandifolia, em manejo antes do plantio, na cultura do feijão (Phaseolus vulgaris). In: CONGRESSO BRASILEIRO DA CIÊNCIA DAS PLANTAS DANINHAS, 21, 1997, Caxambú. Resumos... Caxambú: SBCPD, 1997. p.181.

DEUBER, R. Cultura da soja. In: Ciência das plantas infestantes: manejo. Campinas: Edição do Autor, 1997. v.2. p. 149-165.

EMBRAPA-CNPSo (Londrina, PR). Recomendações técnicas para a cultura da soja no Paraná - 1998/1999. Londrina: 1998. 201p. (Embrapa-CNPSo. Documentos, 119).

MAROCHI, A.I. Avaliação de métodos de controle químico para Richardia brasiliensis (poaia-branca), infestando áreas sob plantio direto da região sul do Brasil. In: Zapp: desafio do novo. São Paulo: Zeneca Agrícola, 1996. p. $175-186$. 
Jamil Constantin et al.

MELHORANÇA, A.L.; CONSTANTIN, J.; PEREIRA, F.A.R. Plantas daninhas. In: Milho: informações técnicas. Dourados: EMBRAPA - Centro de Pesquisa Agropecuária do Oeste: Dourados, 1997, p.112-132. (EMBRAPA-CPAO. Circular Técnica, 5).

PINTO, J.J.O. et al. Manejo de herbicidas dessecantes no sistema de cultivo mínimo na cultura do arroz irrigado. In: CONGRESSO BRASILEIRO DA CIÊNCIA DAS
PLANTAS DANINHAS, 21, 1997, Caxambú. Resumos... Caxambú: SBCPD, 1997. p.165.

PITELLI, R.A. Biologia de plantas daninhas. In: SEMANA DE CONTROLE DE PLANTAS DANINHAS, 10, 1990, Bandeirantes. Anais... Bandeirantes: Fundação Faculdade de Agronomia "Luiz Meneghel", 1990. p.58-100.

RODRIGUES, B.N.; ALMEIDA, F.S. Guia de herbicidas. 4 ed. Londrina: Edição dos Autores, 1998. 648p. 